Cartas al editor

\section{Abuso de tiroxina en un entorno psiquiátrico}

\author{
D. Sloan, M. Hannon y J. O’Boyle \\ Hospital Psiquiátrico de San Vicente, Dublín, Irlanda
}

Una mujer de 35 años de edad con una historia de trastorno afectivo bipolar fue admitida en nuestra unidad psiquiátrica de agudos con rasgos típicos de manía. Estaba irritable, excesivamente locuaz y desinhibida sexualmente. De importancia, le habían diagnosticado hipotiroidismo inducido por litio y había comenzado con $50 \mu \mathrm{g}$ tds de sustitución de tiroxina 4 meses antes. El examen físico fue normal, aparte de una taquicardia en reposo de 100 pulsaciones/min. El examen bioquímico reveló una $\mathrm{T}_{4}$ de $180 \mathrm{nmol} / \mathrm{L}(70-145)$ y una tirotropina (TSH) de $0,2 \mathrm{mIu} / \mathrm{L}(0,3-5,0)$, un patrón consistente con hipertiroidismo. Se descubrió que había abusado de su medicación de tiroxina y, en realidad, había consumido cuarenta tabletas de $50 \mu \mathrm{gm}$ en las 48 horas antes de su admisión. Requirió tratamiento con droperidol intramuscular y haloperidol oral, y se interrumpió la tiroxina. Sus síntomas maniacos disminuyeron en un plazo de 5 días y se le dio de alta. La tiroxina se comenzó de nuevo y la paciente volvió a venir en visita externa.

La asociación entre terapia de litio e hipotiroidismo está bien documentada (Persad et al, 1993), como también lo está la compleja relación entre la perturbación de la homeostasis de la hormona tiroidea y los trastornos afectivos (Gadde et al, 1994). La administración rápida de tiroxina puede causar un aumento brusco en la sensibilidad de las catecolaminas, y se postula que esto, combinado con un aumento de la respuesta de los receptores beta adrenérgicos, es la base neuroquímica para la manía en la tirotoxicosis (Lee et al, 1991). Aunque se ha mostrado antes que la terapia de sustitución de tiroxina para el hipotiroidismo puede precipitar manía en pacientes con trastorno afectivo bipolar (Why- brow, 1994), que sepamos, no se ha comunicado antes abuso de la tiroxina en este entorno. Aunque este fenómeno puede ser muy poco frecuente, se requiere observación clínica perspicaz y pruebas de laboratorio apropiadas para detectar su existencia y proporcionar intervención adecuada.

\section{BIBLIOGRAFIA}

Gadde KM, Krishnan K, Ranga R. Endocrine factors in depresion. Psychiatr Annals 1994; 24: 521-4.

Lee S, Chow CC, Wing YK et al. Mania secondary to thyrotoxicosis. Br J Psychiatry 1991; 159: 712-3.

Persad E, Forbath N, Merskey H. Hyperthyroidism after treatment with lithium. Can J Psychiatry 1993; 38: 599-602.

Whybrow PC. The therapeutic use of triiodothyronine and high dose thyroxine in psychiatric disorder (Review). Acta Medica Austriaca 1994; 21: 47-52.

\section{Priapismo impredecible inducido por neurolépticos: un informe clínico}

M. Razali Salleh ${ }^{1}$, H. Mohamad ${ }^{2}$ y J. Zainol $^{2}$

${ }^{l}$ Departamento de Psiquiatría, ${ }^{2}$ Departamento de Cirugia, Escuela de Ciencias Médicas, Universidad Sains Malaysia, Kelantan, Malasia

El priapismo se define como una erección dolorosa del pene prolongada patológicamente que implica los cuerpos cavernosos, mientras que el cuerpo esponjoso y el glande continúan fláccidos. Por lo general, la erección no está relacionada con excitación o estimulación sexual, aunque algunos casos han ocurrido después de actividad sexual prolongada. En una revisión de las publicaciones, Thompson et al (1990) encontraron que 54 de 261 casos comunicados de priapismo o erección prolongada asocia- 